\title{
Luz ultravioleta para desinfección en áreas de salud, frente al covid-19. Revisión de literatura.
}

\section{Ultraviolet light for disinfection in health areas, in front of covid-19. Literature review.}

\author{
Briones- Cando Natali*1 ${ }^{1}$,Zambrano- Torres Miriam ${ }^{2}$, Febres-Calderón Fabricio ${ }^{3}$,Cuenca-León Katherine ${ }^{4}$ \\ ${ }^{1}$ Rotante de Investigación en la Universidad Católica de Cuenca. Estudiante de pregrado de la Universidad Católica de \\ Cuenca. Cuenca, Ecuador. ORCID iD: https://orcid.org/0000-0003-3716-9080 \\ ${ }^{2}$ Rotante de Investigación en la Universidad Católica de Cuenca, Ecuador. Odontóloga de práctica privada. ORCID iD: \\ https://orcid.org/0000-0002-5789-4696 \\ ${ }^{3}$ Rotante de Investigación en la Universidad Católica de Cuenca, Ecuador. Odontólogo de práctica privada.ORCID iD: \\ https://orcid.org/0000-0001-9126-1297 \\ ${ }^{4}$ Docente Investigadora - Mgs en Análisis biológico y diagnóstico de laboratorio. Universidad Católica de Cuenca. Cuenca, \\ Ecuador. ORCID iD: https://orcid.org/0000-0002-7816-0114 \\ *natubrican@hotmail.com
}

\begin{abstract}
Resumen
La pandemia por el COVID -19 ha provocado una búsqueda acelerada de diferentes técnicas que aseguren la desinfección completa de las áreas de atención hospitalarias y odontológicas. La presente revisión bibliográfica provee al personal de salud información actualizada sobre las diferentes técnicas de desinfección, haciendo especial énfasis en el uso de la luz ultravioleta, su efecto germicida, diferentes espectros de onda y su posible acción ante el virus SARS-CoV 2. Objetivo: Obtener información que respalde el efecto bactericida y viricida de la luz UV, así como establecer los parámetros recomendados para su uso. Discusión: La implementación de luz UV-C como método de inactivación frente a diferentes esporas, bacterias y virus ha tenido un mayor impacto en los últimos meses debido a la nueva realidad a la que los profesionales de la salud se enfrentan. Conclusión: El empleo de la luz UV-C podría reducir significativamente la carga viral en las áreas de la salud evitando infecciones cruzadas al profesional como también al paciente.
\end{abstract}

Palabras clave: Luz ultravioleta; virus, COVID-19; inactivación viral; desinfección.

The COVID -19 pandemic has led to an accelerated search for different techniques to ensure complete disinfection of hospital and dental care areas. This literature review provides health personnel with updated information on the different disinfection techniques, with special emphasis on the use of ultraviolet light, its germicidal effect, different wave spectra and its possible action against the SARS-CoV 2 virus. Objective: To obtain information that supports the bactericidal and viricidal effect of $U V$ light, as well as to establish the recommended parameters for its use. Discussion: The implementation of UV-C light as a method of inactivation against different spores, bacteria and viruses has had a greater impact in recent months due to the new reality that health professionals are facing.Conclusion: The use of UV-C light could significantly reduce the viral load in health areas avoiding cross infections to the professional as well as to the patient.

Key words: Ultraviolet light; virus, COVID-19; virus inactivation; disinfection.

\section{INTRODUCCIÓN}

La pandemia del Covid-19 ha transformado y afectado a nivel mundial los servicios de salud, obligando a encontrar técnicas efectivas para la desinfección de áreas de atención. En esta búsqueda se han desempolvado antiguas estrategias que se encontraban subutilizadas como el uso de luz ultravioleta. ${ }^{1} \mathrm{Ha}$ pasado más de un siglo desde el descubrimiento de su efecto germicida por parte de Niels Finsen y hoy en día se posiciona como la opción de desinfección más prometedora para afrontar la problemática a la que se enfrenta la salud pública en la actualidad. A pesar de su efectividad muchos profesionales no están familiarizados con su uso y existen diversos prejuicios al momento de implementar este sistema por desconocimiento y miedo. ${ }^{2}$ La luz ultravioleta se clasifica en función a su longitud de onda, siendo el rango comprendido entre 200-280 nm (UVC) germicida. El principal 
problema del uso de UVC es el efecto sombra que realizan las diferentes estructuras ubicadas en el área a esterilizar, pero actualmente en el mercado debido a la necesidad de desinfección existen sistemas de luz ultravioleta portables e incluso robots autónomos para la desinfección de áreas de difícil acceso. ${ }^{3}$

El virus SARS-COV2 es un virus nuevo, y actualmente en la literatura existe escasa información de protocolos de uso de luz UV para la inactivación de este virus en específico, pero algunos autores determinan que el uso de la longitud UVC es suficiente para la inactivación de coronavirus, ${ }^{4}$ incluso se han determinado que rangos de onda de entre 200 a 220 um de larga distancia son seguras para su uso en espacios públicos y poseen un efecto germicida muy aceptables. ${ }^{5}$

El objetivo de esta revisión de literatura es proporcionar al personal de salud información científica actualizada acerca de los diversos tipos de sistemas de luz ultravioleta, sus indicaciones, limitaciones y precauciones de uso, para optimizar al máximo esta herramienta de desinfección y que pueda ser adquirida como un complemento para los protocolos de bioseguridad implementados en la consulta.

\section{ESTADO DEL ARTE}

Los coronavirus son conocidos desde hace 50 años, y llamados "corona" por los virólogos debido a la comparación de la corona solar con el exterior del virus. Los primeros reportes de contagios en humanos fueron de 229E y OC43 en 1968. Son una amplia familia de virus con la capacidad de transmisión principalmente en mamíferos, aves y en algunas especies de animales a personas. Compuestos de ARN monocatenarios, con un diámetro de $120 \mathrm{~nm}$, siendo diversos debido a la susceptibilidad a la mutación que ellos presentan. Considerados factores etiológicos para resfriado común y síndromes respiratorios como síndrome respiratorio agudo (SARS-CoV) y el síndrome respiratorio de Oriente Medio (MERS-CoV). 6,7 El mecanismo de acción del COVID-19 es mediado por las glicoproteínas de espiga indispensables para el ingreso a las células huésped teniendo dos subunidades: S1 fijándose a la superficie de la célula del huésped y la S2 adhiriéndose a la membrana celular. Mientras el receptor siendo una enzima convertidora de angiotensina ACE-2 ubicado en la membrana celular se une con la subunidad S1, la serina proteasa transmembrana del huésped (TMPRSS2) activa la espiga interaccionando con la ACE2, y el TMPRSS2 actúa con la subunidad S2, fusionando el virus con la membrana celular y de esta manera permitiendo el ingreso a la célula. ${ }^{6}$

\subsection{Estabilidad de SARS-CoV 2 en las superficies}

Se comparó la estabilidad en aerosoles y diferentes superficies mediante el modelo de regresión bayesiana; se generó este efecto mediante la utilización de nebulizador Collison de tres chorros y mediante un tambor de Goldberg se creó un ambiente en aerosol mediante cinco condiciones ambientales como aerosoles, cartón plástico, cobre y acero inoxidable. ${ }^{9}$ (Tabla 1)

\begin{tabular}{llllll} 
& Aerosoles & Plástico & Acero inoxidable & Cobre & Cartón \\
\hline SARS-CoV-2 & $1.1-1.2$ horas & 6.8 horas & 5.6 horas & $1-2$ horas & $2-4$ horas \\
& & & & & \\
\hline
\end{tabular}

Tabla 1. Vida media de SARS-CoV-2 en las superficies.

\subsection{Características SARS-CoV-2}

Las características que presenta el SARS-CoV 2 se ha visto que es similar a otros virus, sin embargo, su transmisión y niveles de mortalidad se ha visto alto por lo que se llega a considerar que depende de otros factores para que su virulencia sea alta. (Tabla 2$)^{11}$

\begin{tabular}{|c|c|}
\hline Caracteristicas epidemiológicas, clínicas y biológicas. ${ }^{11}$ & SARS-CoV 2 \\
\hline Distribución & Pandemia \\
\hline Origen & Wuhan, provincia de Hubei, China \\
\hline Reservorio Natural & Murciélago* \\
\hline Secuencia viral & $\begin{array}{l}89 \% \text { de similitud con SARS-CoV, } 64 \% \text { con } \\
\text { MERS-CoV }\end{array}$ \\
\hline Probable / sospechoso intermediario anfitión & ¿Los pangolines no conocidos? \\
\hline Transmisión & Extensión de persona a persona Otros ${ }^{\circ}$ \\
\hline Patogenicidad (receptor involucrado) & ACE-2 \\
\hline Número total de personas infectadas en el mundo & Más de 207,800 \\
\hline Período de incubación & 2-14 dias \\
\hline Muertes mundiales & Más de 8,657 \\
\hline Sintomas principales & $\begin{array}{l}\text { Sintomas similares a la gripe, tos seca, } \\
\text { fiebre, diarrea, dolor de cabeza, malestar por } \\
\text { mialgia, falta de aliento. }\end{array}$ \\
\hline Enfermedad letal & Neumonía severa \\
\hline Pacientes con riesgo de muerte o muerte. & $\begin{array}{l}\text { Ancianos y personas con condiciones } \\
\text { preexistentes. }\end{array}$ \\
\hline Rott & 1.4-5.5 \\
\hline Tasa de fatalidad de caso & $05-3 \%$ \\
\hline Niños & Informado con poca frecuencia \\
\hline
\end{tabular}

Tabla 2. Características epidemiológicas, clínicas y biológicas de SARS-CoV 2.

El 31 de diciembre de 2019 en la provincia de Hubei, Wuhan-China se reportó numerosos casos de neumonía de origen idiopático, frecuente en pacientes con edades que redondearon los 55 años de edad y que trabajaban en el mercado mayorista de mariscos de la ciudad. Los síntomas con mayor incidencia era fiebre, tos, mialgia y fatiga siendo fatales en algunos casos. Por lo que en enero del 2020 se indicó sobre la presencia de un nuevo coronavirus como causante de la que se conoce actualmente como COVID-19 nombrado así por el Comité Internacional de Taxonomía de Virus considerándolo como un problema de salud pública mundial debido a la rápida extensión y a las características 
genómicas que presenta. ${ }^{6,10}$ Los casos reportados hasta el 17 de abril de 2020 promediaban 2.074 .529 en más de 150 países confirmando 139.378 muertes.6,10 Según la OMS el 21 de julio de 2020 reporta a nivel global 14.562 .550 casos confirmados de los cuales 607.781 son decesos. ${ }^{8}$

Mientras que una de las zonas más afectadas a nivel mundial por la pandemia de COVID -19 es Latinoamérica, esto se debe a diversos factores tales como precariedad en los sistemas de salud, características sociales como el hacinamiento, pobreza, migración y poca instrucción al personal de salud encargado de la desinfección y tratamiento de superficies de alto contacto. ${ }^{12,13}$ En Ecuador hasta el 21 de julio de 2020 se registró 74.620 casos confirmados por el Ministerio de Salud Pública, pero esta cifra puede ser inferior a la realidad por la baja capacidad de realización de pruebas masivas, zonas de difícil acceso y éxodo humano en zonas fronterizas. ${ }^{13,14} \mathrm{El}$ personal de salud se encuentra en primera línea de defensa contra la pandemia y su riesgo de contagio es inminente. El peligro de una infección aumenta en el sector odontológico que comprende dentistas, higienistas, asistentes dentales y pacientes en general, por su cercanía a la cavidad oral y la presencia de virus en los aerosoles creados por la instrumentación dental. Por este motivo el personal odontológico se ha visto en la necedad de cambiar y mejorar protocolos de desinfección, adecuar los espacios físicos en la consulta y considerar la posibilidad de implementar nuevas estrategias y tecnologías que sean un complemento para la inactivación del virus SARS-COV2. ${ }^{15}$

\subsection{Tipos de mecanismos de inactivación del virus SARS- COV-2 en la consulta odontológica agentes antimicro- bianos}

Actualmente se recomienda el uso de colutorios antes de la atención odontológica para disminuir la carga viral salival del paciente, estudios han demostrado que la utilización de enjuagues de clorhexidina no son suficientes, por lo que es necesario utilizar enjuagues de peróxido de hidrógeno en una concentración del $1 \%$ o enjuagues a base de yodo povidona al $0.2 \% .{ }^{16}$ La desinfección y previa limpieza de las superficies también es de gran importancia, el virus SARSCOV 2 es un virus que posee una envoltura externa y le hace altamente sensible a los agentes desinfectantes como el hipoclorito de sodio $0.1-0.5 \%$, etanol con una concentración del $70 \%$ o glutaraldehído al $2 \% .^{17,18}$

La evidencia que respalda la utilización de dispositivos nuevos para la inactivación del virus SARS-COV 2 todavía es escasa. Pero la necesidad a llevado ha la implementación de nuevos sistemas de aire depurado que permiten filtrar partículas de aire que contengan microorganismos y permiten la recirculación del mismo, al igual que la utilización del aire acondicionado para la ventilación en espacios cerrados siempre y cuando se realice un mantenimiento adecuado. ${ }^{19,20}$ Otros métodos desinfectantes son los gaseosos, el ozono permite una acción superior a los desinfectantes líquidos por su buena dispersión, distribución, penetración y su alto nivel oxidativo. ${ }^{21}$ Sin embargo, el uso de la luz ultravioleta para la desinfección de superficies es una tecnología que ha ganado terreno en estos tiempos por su alta capacidad de eliminar bacterias, virus, esporas, convirtiéndose en un complemento óptimo para la limpieza manual que además es más asequible. ${ }^{22}$

\subsection{Radiación Ultravioleta}

El uso de radiación UV, ha venido mostrando efectividad sobre diversos elementos en especial a ondas cortas cercanas a $\operatorname{los} 240 \mathrm{~nm}$, teniendo en cuenta que se presenta la radiación UVC con un rango de longitud de onda de 200-280 nm, mostrando cambios y respuestas en tejidos vegetales y modificaciones sobre la flora contaminante para el ser humano; es por esta razón que ha sido utilizada sobre el manejo de agua de consumo, así como cárnicos y desactivación de enzimas que modifican la calidad del contenido nutricional de frutas y vegetales. ${ }^{23}$ En el ámbito de desinfección de superficies tiene su efecto sobre la modificación genética celular a partir de procesos oxidativos de sus componentes que permiten la destrucción o separación de los elementos celulares que contienen el material genético transmisible y de esta manera frenar procesos mutagénicos, a partir de cromóforos que serán los que absorban fotones y de esta manera se dan cambios moleculares que serán foto-inducidos desencadenando una señal foto-bioquímica que produce cambios celulares. Entonces se entiende que dentro de los componentes celulares de transferencia genética se encuentra los ácidos nucleicos y elementos proteicos que son cromóforos principales que absorben la radiación UV desde el triptófano y tirosina, pueden darse una serie de daños en la estructura celular principalmente en proteínas y ADN. ${ }^{24}$

\subsection{Clasificación}

La irradiación solar se ha determinado como una gran central termonuclear dirigido a la Tierra pudiendo llegar ondas entre 290 y $5000 \mathrm{~nm}$, esto gracias a la una capa de protección denominada capa de ozono estratosférica, donde se puede encontrar rayos infrarrojos de 800 a $500 \mathrm{~nm}$, luz visible de 400 a $800 \mathrm{~nm}$ y ultravioleta de 190 a $380 \mathrm{~nm} .^{25}$ Teniendo como antecedente que la luz ultravioleta puede ser dañina para el ser humano, esta viene siendo estudiada desde el inicio del análisis de la radiación, conceptualizada por Newton en 1666 presentando su tesis del fenómeno de dispersión de colores haciendo pasar luz por un prisma de vidrio triangular. Rither en 1801 presenta una variación de actividad química, a la que denominó rayos desoxidantes, posterior a esto Maxwell y Hertz elaboran la teoría de que la luz y el sonido son ondas correspondientes a un amplio espectro de energía denominándose ondas electromagnéticas. Los 4 tipos de radiación ultravioleta descritos son: ${ }^{27,28}$ 
- $\quad$ UVV (Radiación ultravioleta al vacío) 100 a 200 nm

- $\quad$ UVC (Germicida) 200 a $280 \mathrm{~nm}$

- UVB 280 - $315 \mathrm{~nm}$

- UVA 315 - $380 \mathrm{~nm}$

Siendo esta UVC la cual tiene menor efecto sobre tejidos, pero una actividad germicida y antivírica, además de la fotoactivación de componentes micronutrientes e inmunoreguladores del organismo como son los P. Leucotomos.26 Entrado en contexto del proceso germicida de la radiación UV, se determinaría la resistencia y tamaño de los organismos sensibles a la radiación. Para obtener un efecto germicida se debe establecer cuál es el cambio molecular existente, destrucción post-exposición y cual es mecanismo para que esto ocurra. ${ }^{26}$

\subsection{Usos y tipos de luz UV-C}

El uso intrahospitalario como técnica y manejo de control y prevención de infecciones adquiridas en centros de atención médica, se da por la aplicación de luz ultravioleta de alta intensidad como lo es la UV-C con una longitud de onda de 200 a $280 \mathrm{~nm}$, siendo un germicida potente para la destrucción del ADN y ARN de bacterias, virus y patógenos evitando que puedan multiplicarse. Para el efecto es necesario conocer cómo se emite este tipo de radiación y cual es su espectro de desinfección; tenemos la luz ultravioleta de Xenón pulsado que desarrolla un destello de luz germicida con una longitud de onda de 200 a 320 nm, este actúa también por reflexión de la luz contra superficies es decir que puede aplicarse en habitaciones hospitalarias donde las superficies son lisas y brillantes. Se encuentran también elementos con mercurio ultravioleta $\mathrm{C}$, que usan bombillas de mercurio a baja presión que emite un espectro de $254 \mathrm{~nm}$, se emplea para bacterias y esporas generando su inactivación genética. ${ }^{27}$

La luz ultravioleta tiene varios efectos en función a su longitud de onda y el efecto mutagénico que este provoca. La UVC es absorbida por las bases de ARN y ADN de los microorganismos por su baja longitud de onda, causando la fusión fotoquímica de dos pirimidinas adyacentes en dímeros unidos covalentemente, que luego se convierten en bases que no se emparejan, evitando así la replicación del microorganismo. ${ }^{28}$ La onda UVB causa la absorción de sus fotones que actúan directamente en la bases de pirimidina como timina, citosina y 5-metilcitosina, también actúa aunque en menor medida en bases de purina, esta acción aunque es similar a la UVC es de 20 a 100 veces menor en cuanto al efecto anti replicante. ${ }^{29}$ La UVA es pobremente absorbida por las células, por lo cual su efecto mutagénico es muy bajo, pero puede causar daño genético adicional a través de la producción de especies reactivas de oxígeno, que causan la oxidación de las bases del material genético de la célula. ${ }^{30}$

Estudios actuales demuestran que para la inactivación de los coronavirus humanos se necesita una longitud de onda de $254 \mathrm{~nm}$, aunque la longitud necesaria podría ser menor ya que los estudios generalmente se realizan en medios in vitro sin considerar la reabsorción de luz que poseen estos materiales. ${ }^{31} \mathrm{Si}$ bien no se han encontrado estudios que determinen la longitud de onda necesaria para la inactivación del virus SARS-CoV 2, Se cree que los lineamientos de inactivación por luz UV para otros coronavirus pueden ser replicables ya que, si bien las mutaciones de ARN pueden tener influencia en su patogenicidad, no brindan cambios estructurales considerables. (Tabla 3$)^{31}$

\begin{tabular}{llll}
\multicolumn{4}{c}{ Longitud de onda para eliminación de coronavirus } \\
\hline Tipo de virus & Longitud de onds $(\mathrm{nm})$ & Medio de estudio & Situación final \\
\hline MERS-COV & 254 & Gotas & Inactivación exitosa \\
\hline SARS-COV1 & 254 & Líquido solución salina & Inactivación exitosa \\
\hline
\end{tabular}

Tabla 3. Longitud de onda para MERS-CoV y SARS-CoV1.

La eficacia de la aplicación de luz UVC dependería del tiempo de exposición que se determine, donde entre 15 a 93 minutos son ideales para inactivar organismos a $254 \mathrm{~nm}$ hasta por aproximadamente 2 metros de su aplicación. ${ }^{32} \mathrm{El}$ rango de los rayos UVC se encuentra entre 200 y $280 \mathrm{~nm}$, estudios demuestran que se puede alcanzar una actividad germicida óptima sin rebasar los $220 \mathrm{~nm}$, sin poner en riesgo la salud del personal, ya que esta longitud de onda puede penetrar microorganismos pequeños ( $<1 \mathrm{um}$ ) pero no es capaz de ingresar al citoplasma de células de mamíferos ni a las del estrato córneo. ${ }^{33,34}$ Una actividad de onda que rebase los 250 $\mathrm{nm}$ puede crear eritema y melanomas, también daños en la retina ,cataratas y tumores oculares, el personal de salud no debe encontrarse en la sala al momento de la desinfección. Actualmente en el mercado existen dispositivos controlados que interrumpen su acción cuando se detecta movimiento en el área a desinfectar. ${ }^{35,36}$

\section{DISCUSIÓN}

Yang G. $2020^{37}$ menciona las vías de transmisión del SARS-CoV 2 son varias siendo la principal entre miembros de familia. Sin embargo, refiere que $3,8 \%$ se produjo entre los trabajadores de la salud por medio de pacientes con COV19 considerando realizar más estudios que defina los mecanismos de transmisión y patogenicidad. Houser K. $2020^{38}$ refiere en un informe dado por el Comité de Fotobiología del IES sobre los efectos de Luz ultravioleta y la inactivación de virus a nivel de ADN como de ARN; considerando la susceptibilidad de estos organismos y la inhibición a la replicación. Deverick J Anderson en un estudio realizado en el año $2017^{39}$ demostró la efectividad de la luz ultravioleta germicida (UVC) cuando se la usa como complemento de desinfectantes tradicionales como las soluciones de hipoclorito, en tratamiento para bacterias multidrogo resistentes 
como la Clostridium difficile, disminuyendo su acción en ambientes hospitalarios.De igual manera Joanne Levin en $2013^{40}$ reportó la disminución de pacientes reinfectados por la mismas bacteria drogo resistente después de la utilización de luz ultravioleta de xenón pulsátil portátil al momento de cambio de cama entre pacientes. Santhi N. $2020^{41}$ mencionó que ante la crisis de implementos de bioseguridad durante la pandemia los trabajadores vulnerando su seguridad por lo que las instituciones de salud se vieron en la obligación de aprovechar las propiedades germicidas de la luz ultravioleta exponiendo los respirador $\mathrm{N} 95$ a dosis de $1 \mathrm{~J} / \mathrm{cm} 2$ con el objetivo de reutilizar dichos implementos si bien indican que no existe una cantidad definida y evidenciada científicamente consideraron como eficaz para mitigar riesgos para los profesionales.

Zambrano $\mathrm{A}$, en un análisis del año $2010^{42}$ señala una reducción del $99 \%$ en el recuento de colonias bacterianas tras 15 minutos de aplicación de radiación UV-C, indicó además una reducción de esporas de C. difficile en $99,8 \%$ con 50 minutos de exposición, definiendo el uso de la radiación UVC aplicada para la destrucción de cadenas de ADN en 200 a $270 \mathrm{~nm}$.La reducción de esporas de C.difficile determinada por Boyce en 2011, ${ }^{43}$ a partir de la aplicación de $254 \mathrm{~nm}$ es comparable con elementos de desinfección de superficies como peróxido de hidrógeno en un periodo de aplicación de la luz de 67.8 minutos para establecer una acción significativa para su reducción.

Esta información coincide con Delgado en $2018^{44}$ que evaluó ocho especies bacterianas formadoras de esporas como Clostridium difficile ribotype 027 y células vegetativas de Staphylococcus aureus, Enterococcus faecium, Escherichia coli y Acinetobacter baumannii entre otras frente a la luz UVC viendo una disminución efectiva por lo que consideró una alternativa para la desinfección en equipos odontológico sin embargo indica que esta técnica requiere una mayor investigación relevantes.

Una manera de limitar las transmisiones virales por aerosoles en el aire es activarlas en poco tiempo después de su producción La literatura es clara en la efectividad de onda de $254 \mathrm{~nm}$ para la eliminación de coronavirus, sin embargo un estudio realizado por Manuela Buonanno, en el año $2020^{45}$ muestra que el uso de onda luz ultravioleta de $220 \mathrm{~nm}$ alejada podría ser la solución para la eliminación de virus en aerosoles en lugares públicos de alta concurrencia.En otro estudio del mismo autor realizado en el año 2016 describe el uso de luz UVC con longitud de onda de $207 \mathrm{~nm}$ produjo resultados que no eran estadísticamente distinguibles a los tratamientos con luz UVC de $254 \mathrm{~nm} .{ }^{46}$ Mientras que un estudio realizado por Marie Lindblad del mismo año ${ }^{47}$ se contrapone a esta postura ya que los en dispositivos de larga distancia si bien los efectos de la onda son menos nocivos no garantizan desinfección en zonas que se encuentran sombreadas y se necesitan estudios de calidad para asegurar una eliminación vírica.
El uso de luz UV-C como método de desinfección frente a diferentes esporas, bacterias y virus ha tenido un mayor impacto en los últimos meses debido a la nueva realidad a la que los profesionales de la salud nos enfrentamos, como se a visto en la recopilación de la literatura varios autores confirman su efecto bactericida y virucida frente a diferente coronavirus existentes siendo esto un antecedente para contrarrestar el virus SARS-CoV 2, sin olvidar que aún se necesita más información que respalde estas afirmaciones.

\section{CONCLUSIONES}

La luz UV-C con efectos germicidas se ha visto como una alternativa, ideal frente a la realidad a la que nos enfrentamos por COVID-19, siendo eficaz en el uso de superficies contaminadas. Varios autores mencionan que la luz UV a 254 nm permiten la destrucción del $99.99 \%$ de microorganismos patógenos. Cabe recalcar que el uso se dirigirá a estructuras inertes mas no en seres humanos debido a los posibles efectos que se podrían generar a nivel ocular, según reportes investigativos.

\section{RECOMENDACIONES}

Se recomienda el uso de luz UV en superficies abióticas, dentro del área de salud y lugares de fluida concurrencia, se debe considerar que la exposición a luz UV para tener una mayor efectividad debe ser total. 
CONFLICTO DE INTERESES: Los autores no 18 Kampf G, Todt D, Pfaender S, Steinmann E. Persistence of manifiestan ningún conflicto de interés.

\section{Referencias Bibliográficas}

1 Mackenzie D. Ultraviolet Light Fights New Virus [published online ahead of print, 2020 Jun 27]. Engineering (Beijing). 2020;doi:10.1016/j.eng.2020.06.009

2 Reed NG. The history of ultraviolet germicidal irradiation for air disinfection. Public health reports.2010;125(1):1527.

3 Ackerman E. Autonomous robots are helping kill coronavirus in hospitals. New York: IEEE Spectrum.2020.

4 Welch D, Buonanno M, Grilj V, et al. Far-UVC light: A new tool to control the spread of airborne-mediated microbial diseases. Sci Rep. 2018;8(1):2752.

5 Welch D, Buonanno M, Shuryak I, et al. Effect of far ultraviolet light emitted from an optical diffuser on methicillin-resistant Staphylococcus aureus in vitro. PLoS One. 2018;13(8):202-275.

6 Hamed MA. Una visión general sobre COVID-19: realidad y expectativa. Bull Natl Res Cent. 2020 junio;44:86.

7 Ministerio de Salud Pública. Dirección de vigilancia epidemiológica. Coronavirus COVID 19.

8 Organización Mundial de la Salud.Informe de situación de la enfermedad por coronavirus 2019 (COVID-19).2020:53 .

9 Van Doremalen N, Morris DH, Holbrook MG, et al. Aerosol and Surface Stability of SARS-CoV-2 as Compared with SARS-CoV-1. NEJM. 2020 March 17.

10 Paudel S, Dangal G, Chalise A, et al. The Coronavirus Pandemic: What Does the Evidence Show. J Nepal Health Res Counc. 2020 Apr 19;18(1):1-9.

11 Contini C, Di Nuzzo M, Barp N, et al. The novel zoonotic COVID-19 pandemic: An expected global health concern. J Infect Dev Ctries. 2020 Mar 31;14(3):254-264.

12 Burki T. COVID-19 in Latin America. Lancet Infect Dis. 2020;20(5):547-548.

13 Villacís JE, López M, Passey D, et al. Efficacy of pulsed-xenon ultraviolet light for disinfection of hightouch surfaces in an Ecuadorian hospital. BMC Infect Dis. 2019;19(1):575.

14 Fiesco-Sepúlveda KY, Serrano-Bermúdez LM. Contributions of Latin American researchers in the understanding of the novel coronavirus outbreak: a literature review. PeerJ. 2020;8: 9332.

15 Checchi V, Bellini P, Bencivenni D., Consolo U. COVID-19 dentistry-related aspects: a literature overview. International dental journal.

16 Peng X, Xu X, Li Y, et al. Transmission routes of 2019$\mathrm{nCoV}$ and controls in dental practice. Int $\mathrm{J}$ Oral Sci. 2020;12(1):9.

17 Morales Navarro D. Acciones del personal de salud del área estomatológica en relación a la COVID-19. Rev Cubana Estomatol. 2020 Mar;57(1): e3245. coronaviruses on inanimate surfaces and their inactivation with biocidal agents. The Journal of hospital infection. 2020;104(3):246-251.

19 Legnani P, Checchi L, Pelliccioni GA, D’Achille C. Atmospheric contamination during dental procedures. Quintessence Int. 1994;25(6):435-439.

20 Leggat PA, Kedjarune U. Bacterial aerosols in the dental clinic: a review. Int Dent J. 2001;51(1):39-44.

21 Martinelli M, Giovannangeli F, Rotunno S, et al. Water and air ozone treatment as an alternative sanitizing technology. J Prev Med Hyg. 2017;58(1):E48-E52.

22 Qureshi Z, Yassin MH. Role of ultraviolet (UV) disinfection in infection control and environmental cleaning. Infect Disord Drug Targets. 2013;13(3):191-195.

23 Quintero-Cerón JP. “Avances en la aplicación de luz ultravioleta de onda corta (UVC) en frutas y vegetales enteros y mínimamente procesados: revisión Advancements in the application of short-wave ultraviolet light (UVC) in whole and fresh-cut fruit and vegetables: a review.”.2013.

24 González-Púmariega M, Tamayo MV, Sánchez-Lamar Á. La radiación ultravioleta. Su efecto dañino y consecuencias para la salud humana. Theoria,2009; 18(2):69-80.

25 Honeyman J. Efectos de las radiaciones ultravioleta en la piel. Rev. Peru. Dermatol.2002;12(2).

26 Vallejo EO, Vargas N., Martínez-Sánchez L., et al. Perspectiva genética de los rayos UV y las nuevas alternativas de protección solar. Revista argentina de dermatología.2003;94.

27 Health Quality Ontario.Portable Ultraviolet Light SurfaceDisinfecting Devices for Prevention of Hospital-Acquired Infections: A Health Technology Assessment. Ont Health Technol Assess Ser. 2018;18(1):1-73.

28 Darnell, M. E, Subbarao, K, Feinstone, S. M, \& Taylor, D. $\mathrm{R}$. Inactivation of the coronavirus that induces severe acute respiratory syndrome, SARS-CoV. Journal of virological methods, 2004;121(1):85-91.

29 Ravanat J, Douki T, Cadet J. Direct and indirect effects of UV radiation on DNA and its components. J Photochem Photobiol B. 2001;63(1-3):88-102.

30 Perdiz D, Grof P, Mezzina M, Nikaido O. Distribution and repair of bipyrimidine photoproducts in solar UV-irradiated mammalian cells. Possible role of Dewar photoproducts in solar mutagenesis. J Biol Chem. 2000;275(35):2673226742.

31 Heßling M, Hönes K, Vatter P, Lingenfelder C. Ultraviolet irradiation doses for coronavirus inactivation - review and analysis of coronavirus photoinactivation studies. GMS Hyg Infect Control. 2020;15.

32 Yang JH, Wu UI, Tai HM, et al.Effectiveness of an ultraviolet-C disinfection system for reduction of healthcare-associated pathogens. Journal of Microbiology, Immunology and Infection.2019; 52(3), 487-493.

33 Buonanno M, Ponnaiya B, Welch D, et al. Germicidal 
efficacy and mammalian skin safety of 222-nm UV light. Radiation research.2017;187(4): 493-501.

34 Woods JA, Evans A, Forbes PD. et al. The effect of 222-nm UVC phototesting on healthy volunteer skin: a pilot study. Photodermatology, photoimmunology \& photomedicine.2015;31(3):159-166.

35 Wengraitis S, Reed NG. Ultraviolet Spectral Reflectance of Ceiling Tiles, and Implications for the Safe Use of UpperRoom Ultraviolet Germicidal Irradiation. Photochemistry and Photobiology.2012; 88(6):1480-1488.

36 Nardell EA, Bucher SJ, Brickner PW, et al. Safety of upper-room ultraviolet germicidal air disinfection for room occupants: results from the Tuberculosis Ultraviolet Shelter Study. Public health reports.2008;123(1):52-60.

37 Guo Y, Cao Q, Hong Z. et al. El origen, la transmisión y las terapias clínicas en el brote de la enfermedad por coronavirus 2019 (COVID-19): una actualización del estado. Military Med Res.2020; 7:11.

38 Houser KW. Ten Facts about UV Radiation and COVID-19, LEUKOS.2020;16(3):177-178.

39 Deverick JA, Luke FC, Weber DL. Enhanced terminal room disinfection and acquisition and infection caused by multidrug resistant organisms and Clostridium difficile (the Benefits of Enhanced Terminal Room Disinfection study): a cluster-randomised,multicentre, crossover study. The lancet.2017.

40 Levin J, Riley LS, Parrish C, et al.. The effect of portable pulsed xenon ultraviolet light after terminal cleaning on hospital-associated Clostridium difficile infection in a community hospital. Am J Infect Control. 2013 Aug;41(8):7468.

41 Narla S, Lyons AB, Kholi I, et al.The importance of the minimum dosage necessary for UVC decontamination of N95 respirators during the COVID-19 pandemic. Whiley. 2020;36(4):324-325.

42 Rutala W, Gergen M, Weber D.Descontaminación ambiental con radiación UV. Rev. chil. infectol. 2010 Dic; 27( 6 ): 573-574

43 Boyce JM, Havill NL, Moore BA. Terminal decontamination of patient rooms using an automated mobile UV light unit. Infect Control Hosp Epidemiol. 2011;32(8):737-742.

44 Delgado D, Ortiz C, Daza H, et al. Evaluación del uso de luz UV como alternativa para la descontaminación de equipos odontológicos. Memorias De Congresos UTP.2018;1(1):4246.

45 Buonanno M, Welch D, Shuryak I,Brenner DJ. Far-UVC light 222nm efficiently and safely inactivates airborne human coronaviruses. Sci Rep. 2020;10(1):10285.

46 Buonanno M, Stanislauskas M, Ponnaiya B, et al. UV light a promising tool for safe low-cost reduction of surgical site infections. II: In-vivo safety studies. PloS one.2016;11(6): e0138418.

47 Lindblad M, Tano E, Lindahl C, Huss F. Ultraviolet-C decontamination of a hospital room: Amount of UV light needed. Burns. 2020;46(4):842-849.

Recibido: 01 de Febrero del 2020

Aceptado: 15 de abril del 2020 
